\title{
Hepatic Inflammatory Pseudotumor-A Diagnostic Challenge: A Case Report and Review of Literature
}

\author{
Ekta Maheshwari ${ }^{1}$ Roopa Ram ${ }^{1}$ Tarun Pandey ${ }^{1}$ Gitanjali Bajaj ${ }^{1}$ Kedar Jambhekar ${ }^{1}$ \\ ${ }^{1}$ Department of Radiology, University of Arkansas for Medical \\ Sciences, Little Rock, Arkansas, United States

\begin{abstract}
Address for correspondence Roopa Ram, MD, UAMS, Department of Radiology, University of Arkansas for Medical Sciences, 4301, West Markham Street, \# 556, Little Rock, AR 72205, United States (e-mail: RRam@uams.edu).
\end{abstract}

J Gastrointestinal Abdominal Radiol ISGAR 2019;2:45-48

\author{
Abstract \\ Keywords \\ - hepatic inflammatory \\ pseudotumor \\ - imaging \\ - malignancy mimic
}

Inflammatory pseudotumor (IPT) is a rare lesion of unknown origin, characterized by proliferation of fibrous connective tissue and invasion of inflammatory cells. Although benign, it has the propensity to mimic malignant masses given its nonspecific symptoms and imaging manifestations. We present a case of hepatic IPT with variable interpretation on different imaging modalities, called as abscess on computed tomography $(\mathrm{CT})$ and neoplasm on ultrasound and magnetic resonance imaging (MRI), thus posing a diagnostic dilemma. Hence it is important to recognize this as a distinct clinical entity and include it in the differential of liver masses with atypical imaging findings.

\section{Introduction}

Inflammatory pseudotumor (IPT) is a rare benign lesion of unknown origin, characterized by proliferation of fibrous connective tissue and invasion of inflammatory cells. Etiology remains uncertain although various theories including infection, autoimmune disease, systemic inflammatory response, trauma, and neoplasm have been postulated. It has been reported at various sites in the body, with the lung and liver being the most common. It is important to recognize this as a distinct entity as it can have a variable appearance on imaging and mimic a malignant mass.

We present a case of hepatic IPT with variable interpretation on different imaging modalities. The lesion was prospectively diagnosed as neoplasm on ultrasound, abscess on computed tomography (CT), and complex cystic neoplasm (biliary cystadenoma) on magnetic resonance imaging (MRI).

\section{Case Presentation}

A 33-year-old African American woman, with no significant past medical history, presented to emergency room with abdominal pain and distension for 6 weeks and fever, chills, nausea, vomiting, and jaundice for 2 weeks.

Review of systems showed no fever or chills and no costophrenic tenderness or shortness of breath with tenderness in the right upper quadrant of the abdomen. There was no weight loss or loss of appetite. Laboratory studies revealed elevated white blood count (WBC) at 21.1 and decreased hemoglobin at 9.2. Neutrophils, lymphocytes, and monocytes were elevated. Serum alkaline phosphatase, bilirubin, and aminotransferases were within normal limits. Serum $\alpha$-fetoprotein was normal.

Ultrasound demonstrated a complex mass in the left hepatic lobe measuring $12.8 \times 6.0 \mathrm{~cm}$ in biaxial dimensions with a heterogenous and echogenic appearance. Vascularity in the septae was seen on color Doppler imaging. Sonographic features were not typical of an abscess ( - Fig. 1) and suggestive of a mixed solid, cystic neoplasm.

To further characterize the lesion, CT of the abdomen and pelvis with oral and intravenous contrast (Omnipaque 350) was performed, and it showed acute noncomplicated diverticulitis of the sigmoid colon. Corresponding to the sonographic abnormality, there was a complex multiloculated lesion in the left lobe of the liver with a mean attenuation value of 42 Hounsfield units on portal-venous phase. The lesion demonstrated peripheral rim enhancement as well as enhancing internal septations ( - Fig. 2A, B). This was interpreted as a liver abscess given the coexisting sigmoid diverticulitis.

MRI was performed on a 1.5 Tesla Phillips magnet with intravenous administration of $13 \mathrm{~mL}$ Dotarem (gadoterate meglumine). It demonstrated a multiloculated, $9.0 \times 3.0 \times 5.7-\mathrm{cm}$ lesion spanning segments II and III that was heterogeneously hyperintense on T2-weighted imaging received

September 19, 2018

DOI https://doi.org/

accepted after revision

October 12, 2018
License terms

()(1) $\Theta \circledast$ 
with scattered cystic areas (-Fig. 3A) and predominantly hypointense on T1-weighted imaging ( - Fig. 3B). Few foci of increased T1 hyperintensity noted within the lesion were presumed to be foci of hemorrhage. Restricted diffusion was noted ( - Fig. 4A, B). Peripheral rim enhancement as well as enhancement of internal septations was noted on administration of intravenous gadolinium with only mild peripheral

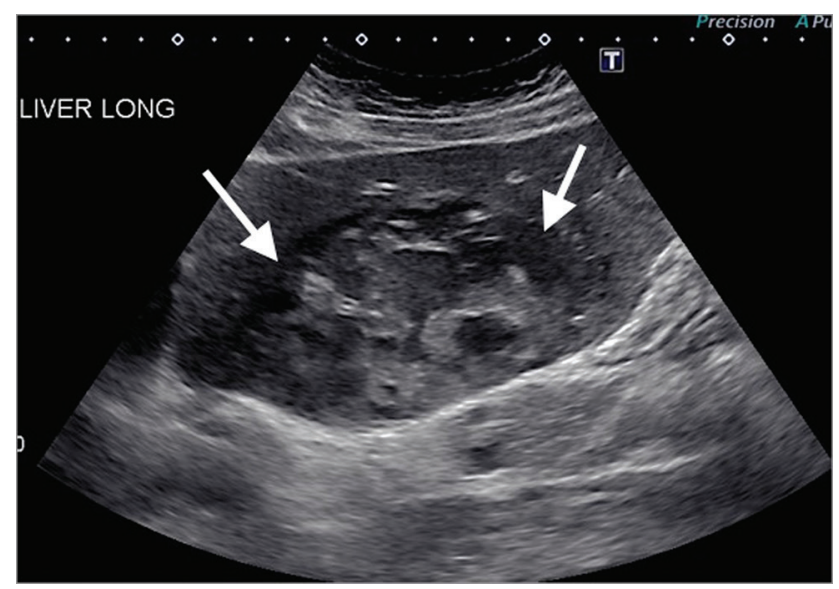

Fig. 1 Ultrasound of liver demonstrates a large oval-shaped mass with well-defined margins in left lobe involving segments II and III with mixed solid and cystic components (arrows).
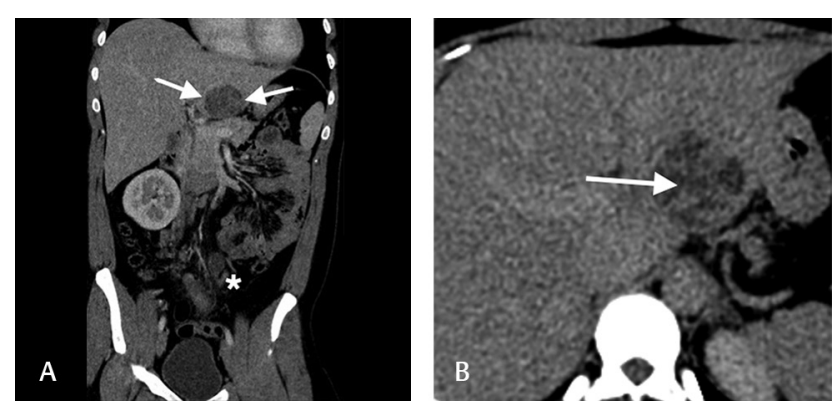

Fig. 2 (A) Computed tomography (CT) of the abdomen and pelvis in portal-venous phase shows a hypodense well-circumscribed mass in left lobe of liver with rim enhancement and mild internal septal enhancement (arrows). Inflamed segment of bowel secondary to diverticulitis in lower abdomen (star). (B) Delayed phase CT scan shows increased enhancement of the septations. hyperemia in the surrounding liver ( $\mathbf{- F i g}$. 5A-C). The internal septae enhanced almost equal to or some even more than the adjacent hepatic parenchyma. Differential diagnosis considered was complex biliary cystic lesion such as a biliary cystadenoma or cystadenocarcinoma rather than an atypical abscess, given the fact that a large pyogenic abscess would elicit marked adjacent hyperemia, entail destruction of hepatic parenchyma, and not demonstrate internal enhancement.

The patient was referred to surgical oncology due to indeterminate etiology and atypical imaging characteristics, and a left hepatectomy was performed. On gross examination, a solid-appearing abnormal area was seen in the left lobe of the liver. Microscopically the abnormality was characterized by thick fibrous septae haphazardly separating regions of foamy as well as pigment-laden macrophages. There was abundant lymphoplasmacytic infiltration. Immunoglobulin G (IgG) stain showed numerous plasma cells, and $\operatorname{IgG} 4$ was positive in only $10 \%$ of the $\operatorname{IgG}$-positive plasma cells (not consistent with IgG4-related disease). Background liver parenchyma surrounding the lesion demonstrated no significant histopathologic change ( - Fig. 6A, B).

\section{Discussion}

IPT is a rare entity, consisting of proliferating fibrous tissue infiltrated by inflammatory cells. It can develop in several organs, such as the brain, spinal cord, thyroid, breast, pancreas, urinary bladder, gastrointestinal tract, and lungs, but it is most common in the lungs and liver. The exact pathogenesis is still unclear, but some studies consider it as a host immunologic response to chronic stimulation by pathogenic microorganisms, neoplasm, pregnancy, or immune system disease. The lesion is often single, consisting mainly of proliferation of connective tissue and invasion of inflammatory cells, often with fat deposition, necrosis, and bleeding. There is a great histologic variation in the composition of IPT reflected in the numerous terms that have been used to describe them, including inflammatory myofibroblastic tumor, plasma cell granuloma, histiocytoma, and fibroxanthoma. ${ }^{1,2}$

Many of the features of IPT can be related to the production of inflammatory mediators such as cytokines and particularly
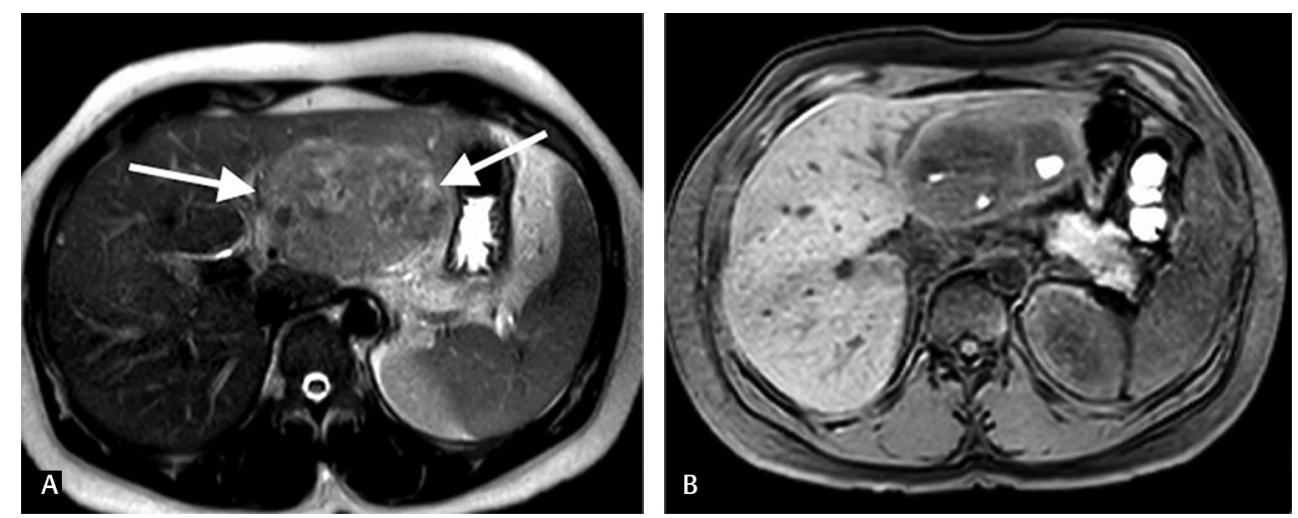

Fig. 3 (A) Axial T2-weighted MRI demonstrates a well-circumscribed hyperintense lesion involving segments II and III of liver with few scattered cystic areas (arrows). (B) Lesion is hypointense on T1-weighted sequences. Few foci of T1 hyperintensity presumed secondary to intralesional hemorrhage. 


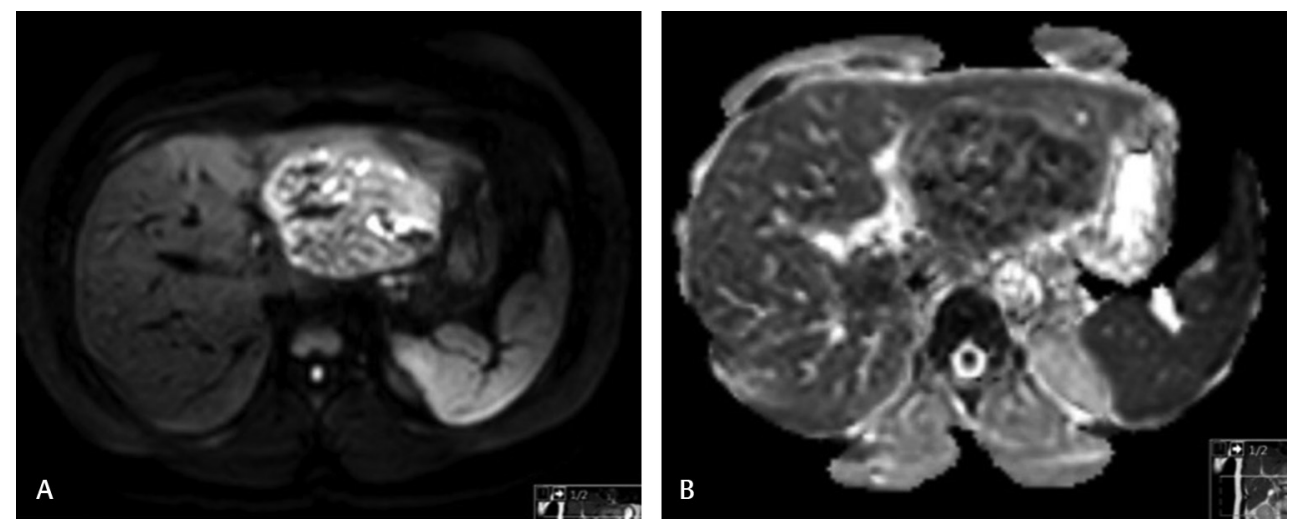

Fig. 4 (A, B) Restricted diffusion noted in the lesion on diffusion-weighted imaging with hyperintense signal on high b-value sequences and hypointensity on corresponding ADC maps.
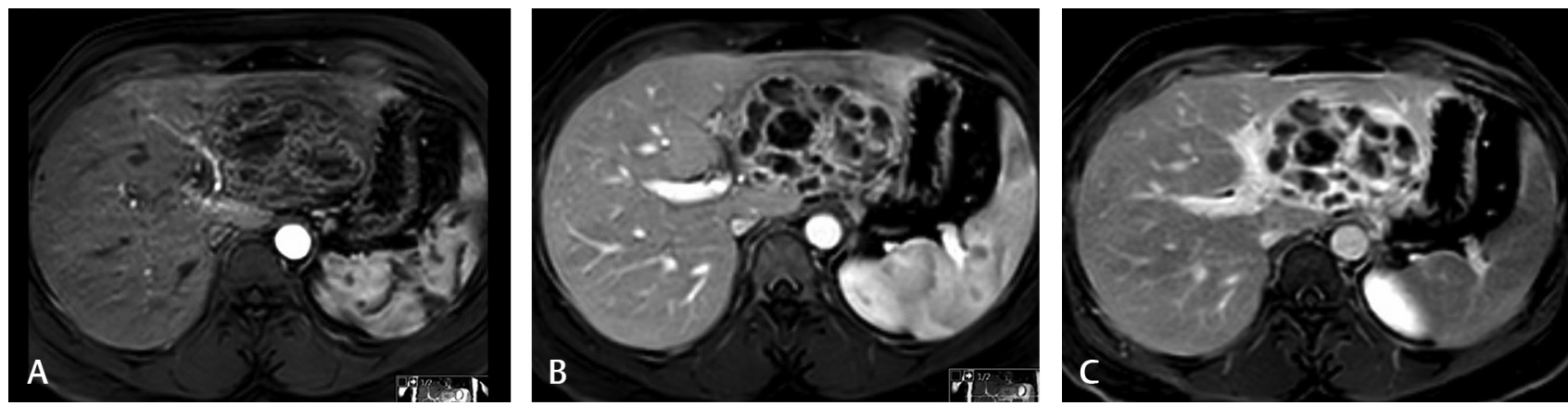

Fig. 5 (A) Postcontrast axial T1-weighted imaging shows mild peripheral and septal enhancement on the arterial phase following administration of intravenous gadolinium. (B, C) Progressive and gradual internal septal enhancement noted on the portal-venous and delayed phases of postcontrast imaging.

interleukin-1. Locally, interleukin-1 stimulates the proliferation of fibroblasts, extravasation of neutrophils, and the activation and increase of procoagulant activity of vascular endothelium. Some authors believe this tumor is a low-grade fibrosarcoma with inflammatory (lymphomatous) cells. It has also been suggested that histiocytic cells predominate in IPTs associated with infection, whereas myofibroblastic cells characterize the lesions more likely to be considered true neoplasms. ${ }^{1}$

IPT of the liver was first described by Pack and Baker in 1953. It is more common in the right lobe of the liver, and though benign, it may mimic malignancy. ${ }^{3}$ Previous studies from Western countries reported that IPT of the liver is common in men of mid-thirties. In contrast, patients from Eastern countries tend to show older age of onset. Males of non-European background are predominantly affected, with a reported male-to-female ratio ranging from 3:1 up to 8:1.

Hepatic IPT is most commonly described as a well-defined and solitary intrahepatic tumor in the right lobe. Multifocal tumors, concurrent hepatic and splenic IPT, and portal vein occlusion have also been reported. Larger lesions may undergo central necrosis. Short interval imaging follow-up may reveal a decrease in the size of lesion suggesting its benignity, ${ }^{4}$ with a few studies also reporting spontaneous regression. ${ }^{5}$

Clinical and laboratory findings are nonspecific, with the most common presenting symptoms being fever, epigastric pain, malaise, and weight loss. Other findings may include obstructive jaundice, hepatomegaly, splenomegaly, and portal hypertension. ${ }^{2}$ Laboratory findings may suggest an inflammatory process with marked leukocytosis, elevated erythrocyte sedimentation rate, and C- reactive protein. Anemia, thrombocytosis, and elevated IgG levels have also been reported. ${ }^{2,4,5}$

On ultrasound, hepatic IPT demonstrates variable echogenicity. Hypoechoic appearance is more common, but hyperechoic and complex lesions have also been described. ${ }^{6}$ Previously, a study using contrast-enhanced ultrasound postulated that pseudotumors demonstrated lack of enhancement in all phases of contrast. A recent study using contrast-enhanced ultrasound has reported that "fast-in and centrifugal-out" pattern of enhancement may be a characteristic pattern in the diagnosis of these lesions. After contrast agent injection, all these lesions showed a fast-in and fast-out enhancement pattern, with hyperenhancement in the early arterial phase and centrifugal washout in the late arterial or early portal-venous phase. The centrifugal washout showed the highest diagnostic ability in differentiating a hepatic IPT from hepatocellular carcinoma, which demonstrates rapid washout with a hypoechoic appearance. $^{7}$

CT findings of IPT are very variable. Hypoattenuating ill-defined masses with varying degrees of contrast enhancement are the most common. Delayed enhancement, especially septal and peripheral, can be seen and is thought to be related to fibrous components. ${ }^{4,5}$

MRI findings of hepatic IPTs are also variable. Hypointense signal relative to the liver on T1-weighted imaging and 

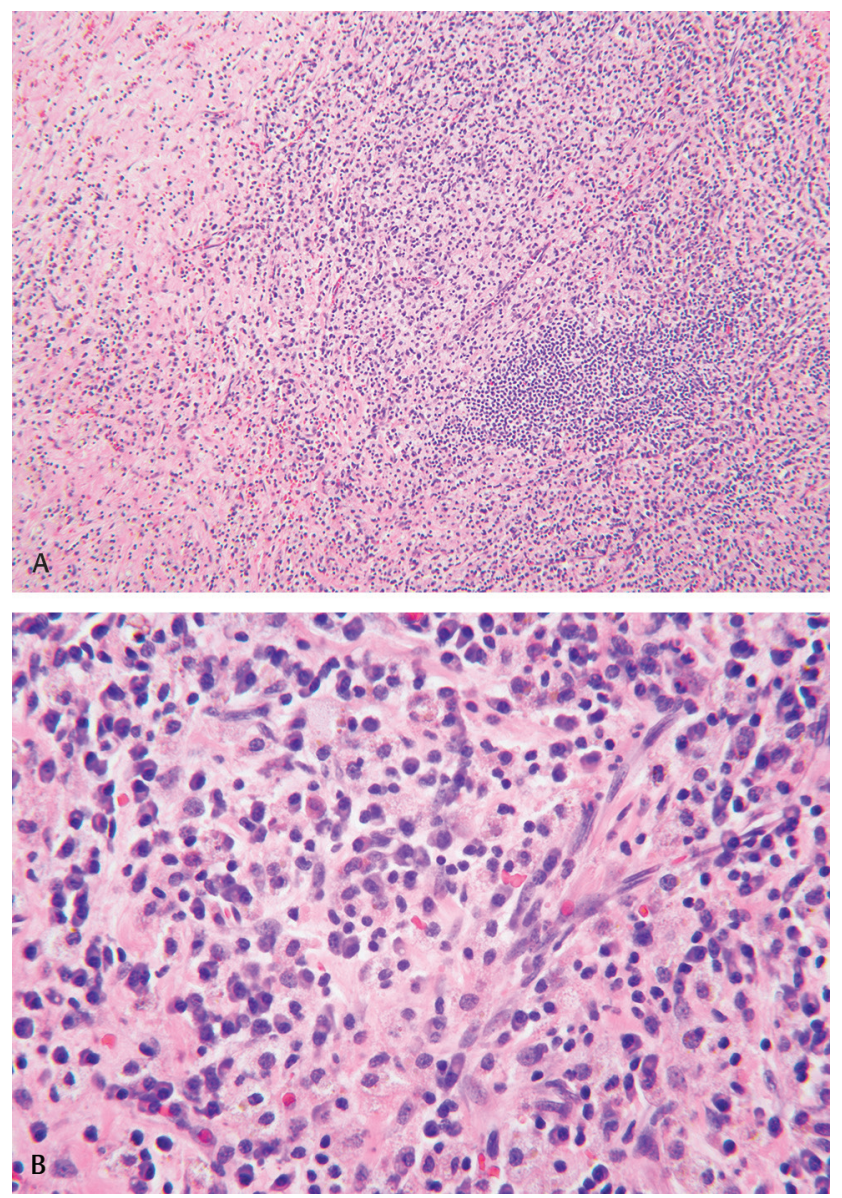

Fig. 6 Two microscopic images from a representative H\&E stain. (A) A $10 \times$ objective (lower power) showing inflammatory cells infiltrating a fibrous stroma. (B) $40 \times$ objective (higher power) highlighting the inflammatory infiltrate that consists predominantly of plasma cells (polytypic by kappa and lambda in situ hybridization stains) and macrophages containing brown yellow pigment. (Image courtesy: Dr. Kari Caradine, Department of Pathology, University of Arkansas for Medical Sciences.)

isointense to hyperintense signal on T2-weighted imaging is common, although iso- and hyperintense lesions on T1 have also been described. ${ }^{5}$ Peripheral and septal enhancements are commonly described after gadolinium administration, secondary to the fibrous components.

Studies that compared the differences of CT and MRI in their diagnostic accuracy for hepatic IPTs concluded that both examination techniques are comparable, and when IPT is being suspected, there is no advantage of combining CT and MRI techniques or using one over the other to obtain a more conclusive diagnosis. They showed an obvious superiority of CT and MRI over ultrasound in terms of diagnostic conclusiveness. ${ }^{8}$

Histopathologic confirmation via percutaneous biopsy or following surgical resection is often necessary to differentiate from other hepatic lesions such as malignant tumors and nonliquefied abscesses. The key to differentiate IPTs from abscesses, especially in the presence of cystic or necrotic components, is the replacement of hepatic parenchyma with collagenous tissue and chronic inflammatory infiltrates in IPT compared with the acute inflammatory infiltrates in an abscess. ${ }^{9}$

Treatment of IPT still remains a controversial point in the literature. In general, IPTs have a benign clinical course.
However, despite their benign nature, some authors have reported their recurrence and potential for malignant transformation..$^{10}$ In the presence of favorable anatomic location and lack of contraindications to surgery, some authors have recommended radical resection of these lesions. Others opine that conservative approaches including antibiotics, nonsteroidal anti-inflammatory drugs, steroids, and biliary drainage should be prioritized. ${ }^{9}$ Hence, the most important step toward correct treatment is accurate diagnosis.

In conclusion, we present a case of hepatic IPT that can mimic both benign and malignant entities. Our case demonstrates the diagnostic challenge of this entity, which has nonspecific imaging findings. In our patient, the presence of a thick-walled multiloculated cystic lesion with concomitant diverticulitis weighted the differential more toward an abscess. Although rare, IPT should be considered in the diagnosis of focal hepatic lesions in a patient with abdominal pain, fever, elevated liver enzymes, and with nonspecific imaging features, especially in the setting of coexisting inflammatory diseases in the abdomen. Histopathologic diagnosis in these cases would be prudent to appropriate management and prevent patients from being subjected to unnecessary surgical intervention.

\section{Funding \\ None.}

\section{Conflict of Interest}

None declared.

\section{References}

1 Narla LD, Newman B, Spottswood S, Narla S, Kolli R. Inflammatory pseudotumor. Radiographics 2003;23 (3):719-729

2 Kawaguchi T, Mochizuki K, Kizu T, et al. Inflammatory pseudotumor of the liver and spleen diagnosed by percutaneous needle biopsy. World J Gastroenterol 2012;18(1):90-95

3 Kim KA, Kim KW, Park SH, et al. Unusual mesenchymal liver tumors in adults: radiologic-pathologic correlation. AJR Am J Roentgenol 2006;187(5):W481-9

4 Nam KJ, Kang HK, Lim JH. Inflammatory pseudotumor of the liver: CT and sonographic findings. AJR A m J Roentgenol 1996;167(2):485-487

5 Yamaguchi J, Sakamoto Y, Sano T, Shimada K, Kosuge T. Spontaneous regression of inflammatory pseudotumor of the liver: report of three cases. Surg Today 2007;37(6):525-529

6 Caramella T, Novellas S, Fournol M, Saint-Paul MC, Bruneton JN, Chevallier P. [Imaging of inflammatory pseudotumors of the liver] [in French]. J Radiol 2007;88(6):882-888

7 Zhang X, Tang S, Huang L, Yu H. Contrast-enhanced sonographic characteristics of hepatic inflammatory pseudotumors. J Ultrasound Med 2016;35(9):2039-2047

8 Yang X, Zhu J, Biskup E, Cai F, Li A. Inflammatory pseudotumors of the liver: experience of 114 cases. Tumour Biol 2015;36(7):5143-5148

9 Tsou YK, Lin CJ, Liu NJ, Lin CC, Lin CH, Lin SM. Inflammatory pseudotumor of the liver: report of eight cases, including three unusual cases, and a literature review. J Gastroenterol Hepatol 2007;22(12):2143-2147

10 Pecorella I, Ciardi A, Memeo L, et al. Inflammatory pseudotumour of the liver-evidence for malignant transformation. Pathol Res Pract 1999;195(2):115-120 